\title{
The Effects of Dislocations on the Crystal Growth Rate of Silicon --A Molecular Dynamics Study
}

\author{
C. ZHANG; B. LIU; K. LI; N. G. ZHOU*; L. ZHOU \\ School of Materials Science and Engineering/Institute of Photovoltaics, Nanchang University, \\ Nanchang 330031, China \\ *Corresponding author: ngzhou@ncu.edu.cn
}

Keywords Dislocation, Crystal Growth Rate, Molecular Dynamics, Silicon

\begin{abstract}
Based on the Tersoff potential, we have investigated the effects of dislocations on the crystal growth rate of Si (110) rough interface by a molecular dynamics simulation. The atomic structures show that, the (110) interface morphology with a $90^{\circ}$ dislocation keeps flatness similar to that of dislocation-free. However, the interface with a $60^{\circ}$ dislocation contains a type of "V" groove, which is formed gradually around the outcrop of the dislocation. The results of the crystal growth rate exhibit that the $60^{\circ}$ dislocation prevents the crystal growth of Si, while the $90^{\circ}$ dislocation has a little influence on the crystal growth rate. The relationship between the dislocation and the crystal growth rate should relate to the difference of interface morphologies.
\end{abstract}

\section{Introduction}

Crystalline silicon solar cells have been occupying the mainstream PV market. The Si crystal grows commonly from melts by directional solidification method. During that grow process, dislocations is nearly inevitable ${ }^{[1]}$. It will lower the photoelectric conversion efficiency. Researchers are devoted to lower the density of dislocations ${ }^{[2]}$. The major techniques is to change the mode of crystal growth and the size of crystal grains, such as mono-like cast silicon, and fine-grain silicon, i.e. high efficiency multicrystalline silicon ${ }^{[3]}$. The crystal growth rate is one key factor of these techniques. Reasonable crystal growth rate could lower the density of dislocations; however, there are few literatures reported how the dislocations to effect crystal growth rate of silicon on the rough interface. According to general theory of crystal growth, dislocations may provide growth steps at the smooth crystal-melt interface, and then affect the crystal growth rate. There should be some connections between the dislocations and crystal growth rate of $\mathrm{Si}$.

In this paper, molecular dynamics simulations of the crystal growth of Si (110) rough interface will be carried out. Three crystal-melt interfaces: dislocation-free, including the end of a $90^{\circ}$ dislocation and including the end of a $60^{\circ}$ dislocation will be investigated. It is expected to find the effect law of dislocations on crystal growth rate of $\mathrm{Si}$ on the rough interface. This law can provide theoretical reference for improving the directional solidification technique and raising the quality of multicrystalline silicon.

\section{Models and Methods \\ Model of Crystal Growth}

The molecular dynamics model of crystal growth is shown in Fig. 1. The Si crystals at both ends of the system represent crystal nuclei for the subsequent crystal growth. While, the Si melt in the middle part of the system are cut from a pre-melting system at high temperature. The dislocation is created basing on the Hirth and Lothe ${ }^{[4]}$ displacement formula, and passes through the part of crystal. The end of dislocation locates at the crystal-melt interface, which is corresponding to (110) plane of the crystal. The whole model contains 150280 atoms, and periodic boundary conditions are applied in three dimensions. The interaction for Si-Si atoms is calculated by Tersoff potential, the system runs in an isothermal-isobaric (NPT) ensemble which is controlled at a standard atmospheric 
pressure and specified temperature by a Nose/Hoover barostat and thermostat. The time step is of $10^{-3} \mathrm{ps}$. In addition, the models of dislocation-free crystal and crystal containing a $60^{\circ}$ dislocation are similar with the model of crystal growth of silicon containing a $90^{\circ}$ dislocation as shown in Fig. 1.

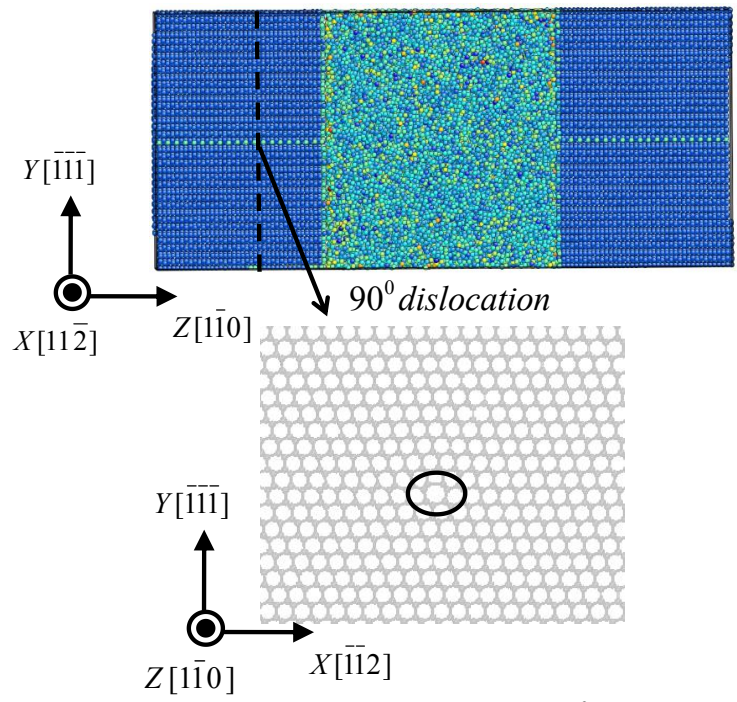

Fig. 1 Molecular dynamics simulation system with a $90^{\circ}$ dislocation for Si (110) Crystal growth from melt

\section{Calculation of Crystal Growth Rate}

Crystal growth rate ${ }^{[5]}$ is calculated by counting the variation of the number of crystal atoms $\left(N_{c}\right)$. To determine whether an atom belongs to the crystal atom or not, we add up the number of "neighbor" of an atom, which are the atoms within the sphere with a radius of average distance of the first and second nearest neighbor distance. If the number of "neighbor" atoms is equal to the value of first nearest neighbor in the corresponding perfect crystal, the atom at the centre of the sphere is a crystal atom, and vice versa. The crystal growth rate can be calculated by the slope of the variation curve of crystal atoms number $\left(N_{c}\right)$ with time, and the formula is as follows:

$$
v(T)=\frac{d_{0}}{N_{0}} \frac{\partial N_{c}(t, T)}{\partial t}
$$

Where $d_{0}$ is the atomic layers spacing and $N_{0}$ represents the number of atoms in each layer.

\section{Results and Discussion}

\section{The Atomic Process of Crystal Growth of Si}

The dislocation-free Si crystal growth process at the reduced temperature of $0.88(\mathrm{~T} / \mathrm{Tm}=0.88)$ is shown in Fig. 2, and each atom is colored by its potential energy. The crystal-melt interfaces keep clear and move gradually into the melt part with the course of time. Finally it is transformed from planar to rough, which is a continuous growth mechanism. The crystal growth processes of Si with dislocations, $60^{\circ}$ dislocations or $90^{\circ}$ dislocations, is similar to that process shown in Fig.2. The differences are the interface morphology and the rate of interface movement, i.e. crystal growth rate.

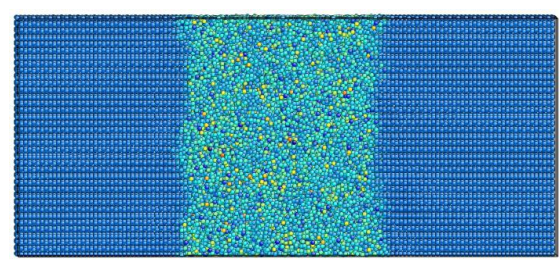


(a) $100 \mathrm{ps}$

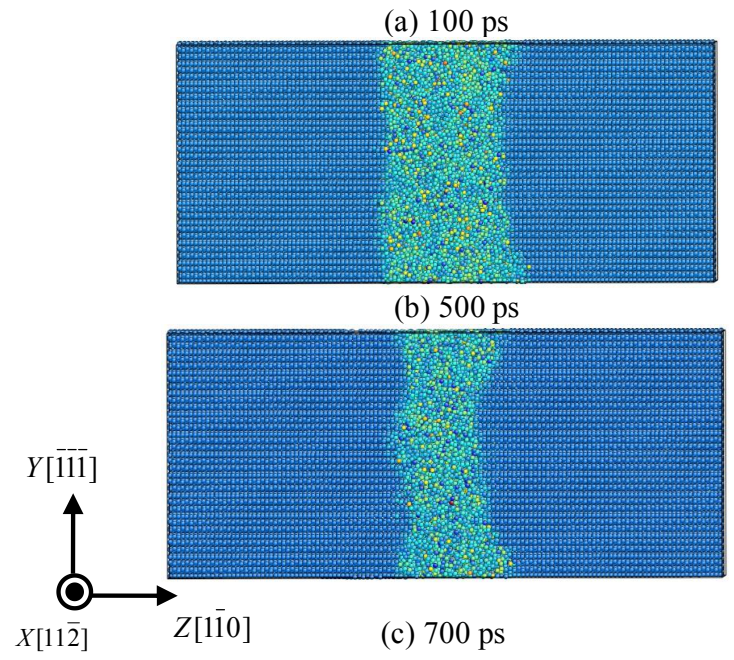

Fig. 2 The atomic structures of the system at different times during the process of crystal growth of $\mathrm{Si}(\mathrm{T} / \mathrm{Tm}=0.88$ ) (a) $100 \mathrm{ps}$ (b) $500 \mathrm{ps}$ (c) $700 \mathrm{ps}$ (The structure for $0 \mathrm{ps}$ is like Fig.1)

\section{Crystal Growth Rate}

Fig. 3 shows the effects of different types of dislocations on the crystal growth rate of Si with the calculation of $N_{c}$. We find the relationship between the crystal growth rate and the reduced temperature is semblable in the three different situations. The results show that, the growth rate first increases fast as the temperature rises, and then decreases slowly after passing through a maximum. The difference is the growth rate of $\mathrm{Si}$ crystal with a $60^{\circ}$ dislocation grows lower than dislocation-free crystal, while the Si crystal with a $90^{\circ}$ dislocation has the approximate growth rate as dislocation-free crystal.

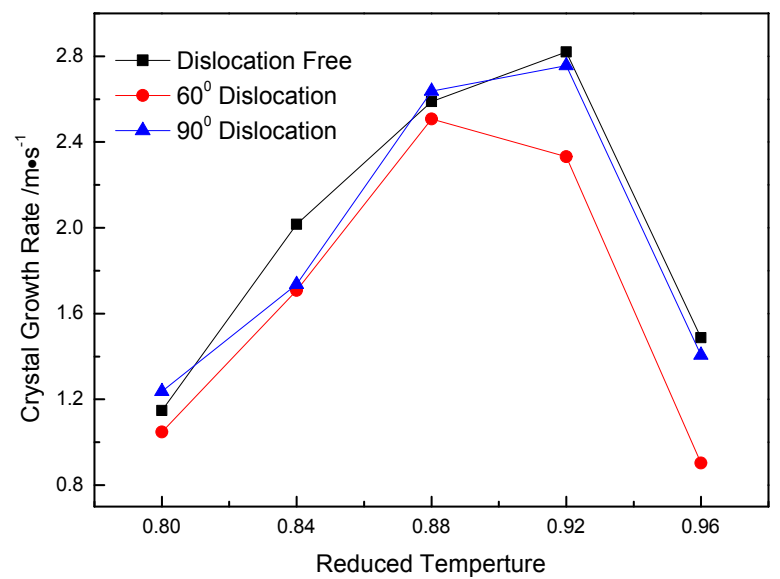

Fig. 3 The growth rate of Si crystal versus the reduced temperature

\section{The Structures of Crystal-Melt Interfaces}

The atomic structure of Si crystal growth with different types of dislocation at 500 ps is shown in Fig. 4. The the interface morphology of dislocation-free crystal has transformed from planar to rough as shown in Fig. 4(a). This rough morphology of Si (110) interface is the similar with the result of situ observation experimentally by Fujiwara ${ }^{[6]}$. Comparing Fig. 4(b) with Fig. 4(a), the morphological transformation of the interface with a $90^{\circ}$ dislocation is little different from that of dislocation-free. It just becomes a little smoother.

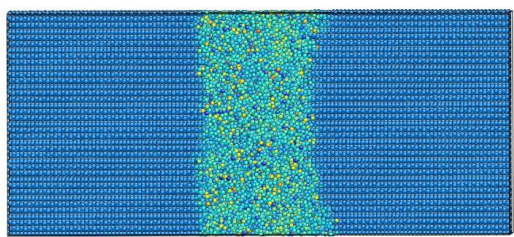


(a) The dislocation-free crystal

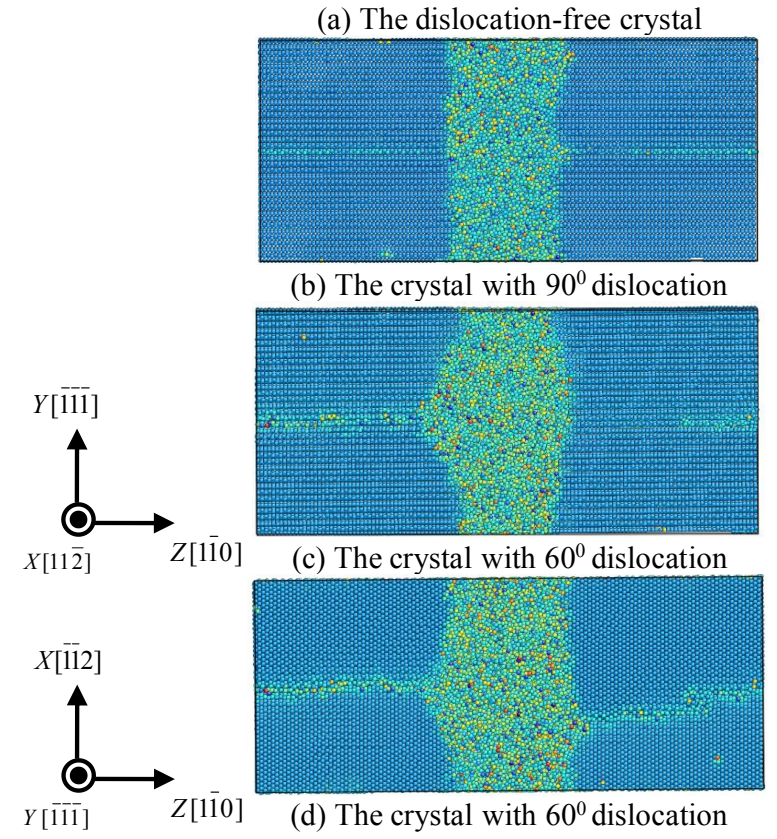

Fig. 4 The atomic structures of the system with different types of dislocation at $500 \mathrm{ps}$ during the simulated growth of Si crystal. (a) The dislocation-free crystal (b) The crystal with $90^{\circ}$ dislocation

(c) The crystal with $60^{\circ}$ dislocation (d) the $90^{\circ}$ rotating picture of (c) by Z[110] direction

Although the outcrop of the $90^{\circ}$ dislocation is more advantage for nucleation on the smooth interface [7, 8], this effect can be negligible because each place is good for nucleation for Si (110) interface, a rough interface. And the crystal grows through a continuous mechanism ${ }^{[9,10,11]}$. This is the reason that there are similar interface morphology and crystal growth rate for the two cases, dislocation-free and a $90^{\circ}$ dislocation.

The morphological transformation of the interface with a $60^{\circ}$ dislocation is markedly different from that of dislocation-free. A type of "V" groove is formed gradually around the outcrop of the $60^{\circ}$ dislocation, as shown in Fig. 4(c) and Fig. 4(d). Fig. 4(d) is the picture of Fig. 4(c) rotated $90^{\circ}$ by $\mathrm{Z}$ [110] direction. It is clearer to show the "V" groove at the right interface where the dislocation has climbed.

An outcrop of a screw dislocation can mostly emit growth spirals, and the crystal grows through a spiral step mechanism [12, 13]. However, the crystal growth rate of the growth mechanism depending on screw dislocation steps is lower than that of continuous mechanism under a certain range of the driving force ${ }^{[14]}$. The $60^{\circ}$ dislocation is a type of mixed dislocation, which consists of edge dislocation component and screw dislocation component. The "V" groove of the $60^{\circ}$ dislocation is inclined to provide spiral-like steps $\left.{ }^{[15,16}\right]$. It indicates that the crystal growth is partial of the growth mechanism depending on screw dislocation steps, so the "V" groove of the $60^{\circ}$ dislocation will lower the growth rate as shown in Fig. 3.

\section{Conclusions}

The crystal growth of Si (110) plane has been simulated by molecular dynamics. The crystal growth rate and the morphology of crystal-melt interface have been compared among the interface with different dislocations. The results show that, there is little influence of $90^{\circ}$ dislocations on the crystal growth of Si (110). The crystal growth rate and the morphology of the interface with a $90^{\circ}$ dislocation are similar to that of dislocation-free. However, The $60^{\circ}$ dislocation lowered the crystal growth rate of Si (110), and a type of "V" groove is formed gradually around the outcrop of the dislocation during crystal growth. 


\section{Acknowledgement}

This research was financially supported by the National Science Foundation (No. 51361022, No. 51561022 and No.5126403) and the Natural Science Foundation of Jiangxi Province (No. 20151BAB206001).

\section{References}

[1] Ryningen, B., Stokkan, G., Kivambe, M., Ervik, T. \& Lohne, O., Growth of dislocation clusters during directional solidification of multicrystalline silicon ingots. Acta Materialia, 59(20), pp. 7703-7710, 2011

[2] Jouini, A., Ponthenier, D., Lignier, H., Enjalbert, N., Marie, B., Drevet, B. \& Camel, D., Improved multicrystalline silicon ingot crystal quality through seed growth for high efficiency solar cells. Progress in photovoltaics: research and applications, 20(6), pp. 735-746, 2012.

[3] Zhu, D., Ming, L., Huang, M., Zhang, Z. \& Huang, X., Seed-assisted growth of high-quality multi-crystalline silicon in directional solidification. Journal of Crystal Growth, 386, pp. 52-56, 2014.

[4] Hirth, J. P. \& Lothe, J., Theory of Dislocations. Second edition. Wiley, 1982.

[5] Ding, F., Bolton, K., Rosen, A., Molecular dynamics study of the surface melting of iron clusters. The European Physical Journal D-Atomic, Molecular, Optical and Plasma Physics, 34(1-3), pp. 275-277, 2005.

[6] Fujiwara, K., Gotoh, R., Yang, X. B., Koizumi, H., Nozawa, J., \& Uda, S., Morphological transformation of a crystal-melt interface during unidirectional growth of silicon. Acta Materialia, 59(11), pp. 4700-4708, 2011.

[7] Frank, F.C., J. Crystal Growth, 51, pp. 367, 1981.

[8] Giling, L.J., \& Dam, B., A rough heart model for edge dislocations which act as persistent growth sources. Journal of Crystal Growth, 67(2), pp. 400-403, 1984.

[9] Jackson, K.A., Liquid metal and solidification. ASM, Cleveland, pp. 174, 1958.

[10]Jackson, K.A., Crystal growth kinetics. Materials science and engineering, 65(1), pp. 7-13, 1984.

[11] Abraham, F.F. \& Broughton, J.Q., Pulsed melting of silicon (111) and (100) surfaces simulated by molecular dynamics. Physical review letters, 56(7), pp. 734,1986.

[12] Frank, F.C., Disc. Faraday Society, 5, pp. 48, 1949.

[13] Wang, Q.D., Ding, W.J., \& Jin, J.Z., Step growth of a primary silicon crystal observed by decantation during centrifugal casting. Materials science and technology, 8(13), pp. 921-925, 2013.

[14] Que, D.L., Silicon materials science and technology. Zhejiang: Zhejiang University press, pp. 120, 2000. (In Chinese)

[15] Frank F.C., Capillary equilibria of dislocated crystals. Acta Crystallographica, 4(6), pp. 497-501, 1951.

[16] Sunagawa, I. \& Bennema, P., Observations of the influence of stress fields on the shape of growth and dissolution spiral. Journal of Crystal Growth, 53, pp. 490-504, 1981. 